\title{
Influence of centrifugation conditions on the results of 77 routine clinical chemistry analytes using standard vacuum blood collection tubes and the new BD-Barricor tubes
}

Janne Cadamuro*1, Cornelia Mrazek1, Alexander B. Leichtle², Ulrike Kipman³ ${ }^{3}$ Thomas K. Felder ${ }^{1}$, Helmut Wiedemann', Hannes Oberkofler ${ }^{1}$, Georg M. Fiedler², Elisabeth Haschke-Becher ${ }^{1}$

\author{
${ }^{1}$ Department of Laboratory Medicine, Paracelsus Medical University, Salzburg, Austria \\ 2University Institute of Clinical Chemistry, Inselspital, Bern University Hospital, University of Bern, Switzerland \\ ${ }^{3}$ UT SPSS Statistics, Hallein, Austria
}

*Corresponding author: j.cadamuro@salk.at

\begin{abstract}
Introduction: Although centrifugation is performed in almost every blood sample, recommendations on duration and g-force are heterogeneous and mostly based on expert opinions. In order to unify this step in a fully automated laboratory, we aimed to evaluate different centrifugation settings and their influence on the results of routine clinical chemistry analytes.

Materials and methods: We collected blood from 41 healthy volunteers into BD Vacutainer PST II-heparin-gel- (LiHepGel), BD Vacutainer SST IIserum-, and BD Vacutainer Barricor heparin-tubes with a mechanical separator (LiHepBar). Tubes were centrifuged at 2000xg for 10 minutes and $3000 \mathrm{xg}$ for 7 and 5 minutes, respectively. Subsequently 60 and 21 clinical chemistry analytes were measured in plasma and serum samples, respectively, using a Roche COBAS instrument.

Results: High sensitive Troponin T, pregnancy-associated plasma protein A, B human chorionic gonadotropin and rheumatoid factor had to be excluded from statistical evaluation as many of the respective results were below the measuring range. Except of free haemoglobin (fHb) measurements, no analyte result was altered by the use of shorter centrifugation times at higher $\mathrm{g}$-forces. Comparing LiHepBar to LiHepGel tubes at different centrifugation setting, we found higher lactate-dehydrogenase $(L D)(P=0.003$ to $<0.001)$ and lower bicarbonate values $(P=0.049$ to 0.008$)$ in the latter.

Conclusions: Serum and heparin samples may be centrifuged at higher speed (3000xg) for a shorter amount of time (5 minutes) without alteration of the analytes tested in this study. When using LiHepBar tubes for blood collection, a separate LD reference value might be needed.
\end{abstract}

Key words: pre-analytics; centrifugation; diagnostic tests; routine

\section{Introduction}

Apart from sample collection and transportation, sample preparation by centrifugation is one of the most critical steps in the pre-analytical phase. In order to obtain the best sample material quality for laboratory analysis centrifugation time, speed and temperature are crucial. Prolonged centrifugation at high speed might lead to haemolysis or structural damage to the measurand whereas brief low speed centrifugations may lead to insufficient separation of plasma or serum from cellular blood components (1). As a consequence, laboratory analyses might be altered due to chromatic interferences, loss of analytes being metabolized or consumed and interference by residual cellular components such as platelets, leucocytes or their components (2-5). In addition to these quality issues of sample preparation, many if not all laboratories have to keep turnaround times (TATs) in the total testing process as short as possible in order to rapidly diagnose the patient. As the duration for 
the analytical process usually is invariably determined by the respective analytical device, reducing the TAT within the laboratory may be achieved by optimizing the centrifugation conditions. One obstacle in such optimization attempts is the fact that these conditions depend on the type of sample or even on the type of separator. For example, coagulation tubes should usually be centrifuged for a longer period of time than samples for clinical chemistry analyses to retrieve platelet poor or even platelet "free" plasma (6). In current recommendations on sample centrifugation, there is a big variety in terms of centrifugation time, speed and temperature, ranging from 10 to 20 minutes and 1500 to $3000 \times$ at 15 to $25^{\circ} \mathrm{C}$, respectively (613). These parameters also change depending on the gel composition used in the specific country with g-forces as low as 1000xg (14). The manufacturer of the newly introduced BD-Barricor tube, which uses a mechanical separator instead of a gel barrier, claims that these tubes may be centrifuged at higher speed and shorter time, recommending centrifugation for 5 minutes at $3000 x g$ or only 3 minutes at $4000 \mathrm{xg}$.

Often it is referred to the centrifugation recommendation of blood collection tube manufacturers (15). However, picturing the number of possible laboratory parameters on all of the possible analytical platforms, it seems unlikely that providers of blood collection tubes are able to validate every single one of these combinations. Even if such a validation would be available, tube manufacturers would have to find a way of validating also newly introduced parameters measured in plasma or serum, collected into one of their tubes. Therefore tube vendors most probably recommend to centrifuge for a longer duration than necessary to prevent incomplete plasma/serum separation and to cover most if not all possible analytical combinations (11-13). We therefore believe that manufacturers of analytical devices or assays should validate sample preparation settings for their products, taking tube characteristics in terms of material or resistance to temperature and gforces into account, instead of relying on recommendations of tube manufacturers. However, these validation data are often lacking.
In our hospital Vacutainer, LH PST ${ }^{\text {TM }}$ II lithium heparin and Vacutainer, SST ${ }^{\mathrm{TM}}$ II Advance serum tubes as well as the Vacutainer Barricor ${ }^{\top \mathrm{M}}$ tubes (all tubes from Becton Dickinson, Franklin Lakes, NJ/USA), which are used in our emergency department, are centrifuged for 10 minutes at $2000 \mathrm{xg}$. As we aim to implement fully automated pre-analytical and analytical processes, we were seeking to achieve a unified and standardized sample centrifugation with the shortest time possible for most if not all sample types without significantly altering plas$\mathrm{ma} / \mathrm{serum}$ quality. In the field of haemostasis, respective evaluations of shortened centrifugation time at higher $\mathrm{g}$-forces are available in recent literature (3,16-18). For clinical chemistry parameters however, these data are scarce (19).

In this study we therefore aimed to evaluate a standardized sample preparation protocol for clinical chemistry analyses by comparing different centrifugation conditions in heparin-gel-, serumand Barricor tubes.

\section{Materials and methods}

This study was conducted at the Department of Laboratory Medicine and Microbiology of the University Hospital Salzburg between August and November 2016.

After approval by the local ethics committee (Protocol number 415-E/2028/9-2016) and a written informed consent of every participant, three Lithium-Heparin gel tubes (LiHepGel) (BD Vacutainer, LH PST TM II, 16x100mm, 8ml REF:367378), three serum gel tubes (BD Vacutainer, SST ${ }^{\mathrm{TM}}$ II Advance, 16x100mm, 8ml REF: 367953) and two Lithium Heparin tubes using a mechanical separator ( $\mathrm{Li}-$ HepBar) (BD Vacutainer Barricor LH Plasma, 13x100mm, 5ml REF: 365039) were drawn from 41 healthy volunteers ( 17 males and 24 females with a median (range) age of 42 (20-74) years) according to current recommendations (20). All blood collections were performed using a $21 \mathrm{G}$ winged safety blood collection set (GBO Vacuette safety blood collection set, $19 \mathrm{~cm}$, REF 450081; Greiner BioOne, Kremsmünster, Austria). The order of sample tube collection was randomized in every participant 
and the tourniquet was removed while the first tube was filled. All samples were mixed according to the manufacturer's instructions. LiHepGel and LiHepBar tubes were processed immediately after sample collection. Serum samples were stored at room temperature in an upright position for 30 minutes to allow the sample to clot appropriately before further processing (21). Always one of the respective LiHepGel and serum tubes were centrifuged at $2000 \mathrm{xg}$ for 10 minutes, as well as at $3000 x g$ for 7 minutes and at 3000xg for 5 minutes. The two LiHepBar tubes were centrifuged at 2000xg for 10 minutes and at 3000xg for 5 minutes (Figure 1). There was no LiHepBar tube being centrifuged at $3000 \mathrm{xg}$ for 7 minutes as recommendations emphasize an even shorter time of 5 minutes at this g-force. High throughput centrifuges as used in our laboratory mostly reach a maximum of about $3000 \mathrm{xg}$, therefore no higher g-forces were evaluated. All centrifugations were performed on Hettich centrifuges (Rotanta 460R and Rotixa 500RS, Hettich Lab Technology, Tuttlingen, Germany) using a swing-out rotor at a temperature of $22^{\circ} \mathrm{C}$. Sixty analytes were measured in heparin plasma and another 21 analytes were measured in serum. Analyses for all of the 81 parameters, measurable on the Roche COBAS 8000 device (Roche Diagnostics, Rotkreuz, Switzerland) were performed in duplicate, immediately after centrifugation (Table 1).

Twice daily quality controls (QC) controls within the target range and Westgard rules $\left(1_{3 s^{\prime}}, 1_{2 s^{\prime}} 2_{2 s^{\prime}}\right.$ $\mathrm{R}_{4 \mathrm{~s}}$ and $2 \mathrm{of} 3_{2 \mathrm{~s}}$ ) were a premise for analyses of the samples. Control samples from two different manufacturers were used (Roche Diagnostics, Basel, Switzerland and Bio-Rad Laboratories, Hercules, USA). Coefficients of variations (CV) for QC of all analytes tested are shown in Table 1 and Table 2. For calculation of these CVs we used data from the current lot of the low level control of respective parameters. If this lot was in use for less than two weeks, we additionally used the data of the previous lot. Haemolysis index (HI) measurements are referred to as free haemoglobin ( $\mathrm{fHb}$ ), since respective measurements from this instrument are directly correlated with an $\mathrm{HI}$ of 1 being equal to a $\mathrm{fHb}$ value of $0.01 \mathrm{~g} / \mathrm{L}(22,23)$. If test results were be-
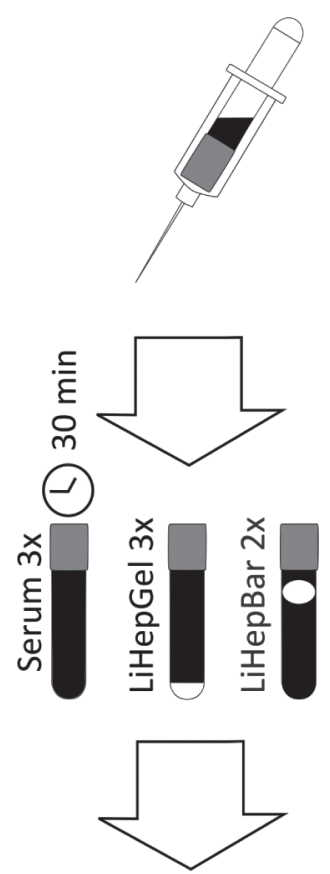

()

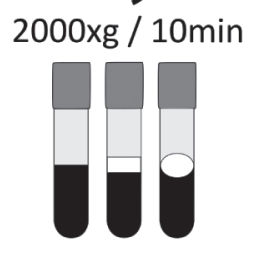

(ด) $3000 \times \mathrm{g} / 7 \mathrm{~min}$
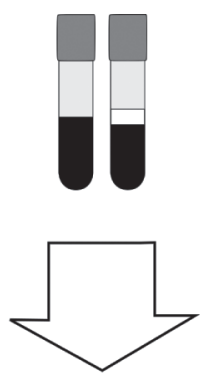

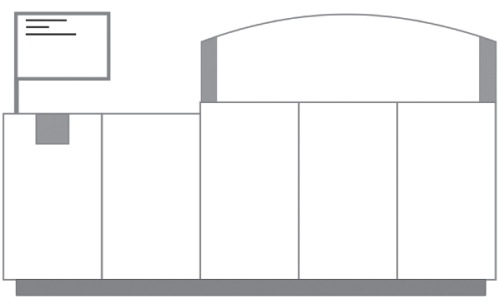

Figure 1. Sample collection and preparation workflow. LiHepGel - vacuum lithium heparin gel tubes. LiHepBar - vacuum lithium heparin Barricor tubes.

low the measurement range, all according values of the other centrifugation settings from this specific sample were not considered for further analysis. If results from more than $50 \%$ of samples had to be excluded, the respective analyte was eliminated from statistical analyses. 
TABLE 1. Comparison of laboratory values measured in plasma for different centrifugation conditions and tube type

\begin{tabular}{|c|c|c|c|c|c|c|c|c|}
\hline \multirow{3}{*}{ Parameter, unit } & \multirow{3}{*}{$\mathbf{N}^{*}$} & \multirow{3}{*}{ CV (\%) $)^{\dagger}$} & \multicolumn{5}{|c|}{ Centrifugation conditions per tube type } & \multirow{3}{*}{$\mathbf{P}$} \\
\hline & & & $2000 \times \mathrm{xg} / 10 \mathrm{~min}$ & $2000 \times \mathrm{xg} / 10 \mathrm{~min}$ & $3000 \times g / 7 m i n$ & $3000 \times \mathrm{xg} / 5 \mathrm{~min}$ & $3000 \times g / 5 \mathrm{~min}$ & \\
\hline & & & LiHepGel & LiHepBar & LiHepGel & LiHepGel & LiHepBar & \\
\hline Chloride, $\mathrm{mmol} / \mathrm{L}$ & 41 & 1.6 & $98(2)$ & $98(3)$ & $98(3)$ & $98(3)$ & $98(3)$ & 1.0 \\
\hline Bicarbonate, mmol/L & 41 & 11.1 & $28(2)$ & $29(2)$ & $28(2)$ & $28(2)$ & $28(2)$ & 0.286 \\
\hline Potassium, $\mathrm{mmol} / \mathrm{L}$ & 41 & 1.1 & $4(0.3)$ & $4(0.2)$ & $4(0.3)$ & $4(0.3)$ & $4(0.3)$ & 1.0 \\
\hline Sodium, $\mathrm{mmol} / \mathrm{L}$ & 41 & 1.0 & $138(3)$ & $138(2)$ & $138(2)$ & $138(2)$ & $138(2)$ & 1.0 \\
\hline Urea, $\mathrm{mmol} / \mathrm{L}$ & 41 & 18.9 & $4(2)$ & $4(2)$ & $4(2.2)$ & $4(2)$ & $4(2)$ & 1.0 \\
\hline Creatinine, $\mu \mathrm{mol} / \mathrm{L}$ & 41 & 6.3 & $71(22.1)$ & $71(26.6)$ & $71(22.1)$ & $71(17.7)$ & $71(26.6)$ & 1.0 \\
\hline $\begin{array}{l}\text { Creatinine estimated } \\
\text { glomerular filtration rate, } \\
\mathrm{mL} / \mathrm{min} / 1.73 \mathrm{~m}^{2}\end{array}$ & 41 & / & $94(16)$ & $95(17)$ & $95(16)$ & $95(16)$ & $96(16)$ & 1.0 \\
\hline Calcium, mmol/L & 41 & 5.0 & $2.32(0.14)$ & $2.31(0.11)$ & $2.32(0.12)$ & $2.32(0.11)$ & $2.31(0.13)$ & 1.0 \\
\hline Total protein, g/L & 41 & 4.6 & $73(6)$ & $74(6)$ & $73(7)$ & $74(5)$ & $73(6)$ & 1.0 \\
\hline Glucose, mmol/L & 41 & 4.8 & $94(11)$ & $93(12)$ & $94(10)$ & $93(11)$ & $93(9)$ & 1.0 \\
\hline C-reactive protein, $\mathrm{mg} / \mathrm{L}$ & $24(17)$ & 5.5 & $2(3.25)$ & $2(3.25)$ & $2(3.25)$ & $2(3.25)$ & $2(3.25)$ & 1.0 \\
\hline $\begin{array}{l}\text { High sensitive C-reactive } \\
\text { protein, } \mathrm{mg} / \mathrm{L}\end{array}$ & 41 & 8.7 & $0.9(1.4)$ & $0.9(1.5)$ & $0.9(1.4)$ & $0.9(1.6)$ & $0.9(1.5)$ & 1.0 \\
\hline Uric acid, $\mathrm{mmol} / \mathrm{L}$ & 41 & 4.9 & $0.29(0.12)$ & $0.29(0.12)$ & $0.29(0.11)$ & $0.29(0.11)$ & $0.29(0.11)$ & 1.0 \\
\hline Phosphorus, mmol/L & 41 & 6.5 & $0.97(0.22)$ & $0.97(0.24)$ & $0.96(0.22)$ & $0.98(0.22)$ & $0.97(0.21)$ & 1.0 \\
\hline Magnesium, mmol/L & 41 & 5.5 & $0.82(0.07)$ & $0.82(0.07)$ & $0.82(0.07)$ & $0.83(0.06)$ & $0.82(0.06)$ & 1.0 \\
\hline Bilirubin total, $\mu \mathrm{mol} / \mathrm{L}$ & 41 & 4.0 & $8.6(5.1)$ & $8.6(5.1)$ & $8.6(6.8)$ & $8.6(5.1)$ & $8.6(5.1)$ & 1.0 \\
\hline Bilirubin direct, $\mu \mathrm{mol} / \mathrm{L}$ & 41 & 5.8 & $3.4(2.4)$ & $3.4(0.7)$ & $3.4(0.7)$ & $3.4(0.9)$ & $3.4(0.9)$ & 1.0 \\
\hline Cholesterol, $\mathrm{mmol} / \mathrm{L}$ & 41 & 5.8 & $5(1.1)$ & $5(1.1)$ & $5(1.2)$ & $4.9(1.1)$ & $5(1)$ & 1.0 \\
\hline Triglycerides, $\mathrm{mmol} / \mathrm{L}$ & 41 & 4.8 & $1(0.6)$ & $1(0.6)$ & $1.1(0.6)$ & $1(0.6)$ & $1.1(0.6)$ & 1.0 \\
\hline HDL-Cholesterol, $\mathrm{mmol} / \mathrm{L}$ & 41 & 6.2 & $1.8(0.6)$ & $1.8(0.6)$ & $1.8(0.6)$ & $1.8(0.6)$ & $1.9(0.6)$ & 1.0 \\
\hline Apo-Lipoprotein B, mg/L & 41 & 3.9 & $940(340)$ & $940(320)$ & $950(310)$ & $930(320)$ & $940(310)$ & 1.0 \\
\hline Lipoprotein (a), nmol/L & $32(9)$ & 4.0 & $27.8(90)$ & $28.8(91.4)$ & $28.2(88.9)$ & $27.8(90.1)$ & $28.4(91.7)$ & 1.0 \\
\hline $\begin{array}{l}\text { Aspartate-transaminase, } \\
\mathrm{U} / \mathrm{L}\end{array}$ & 41 & 5.6 & $21(8)$ & $20(9)$ & $22(8)$ & $22(9)$ & $22(7)$ & 1.0 \\
\hline $\begin{array}{l}\text { Alanine-transaminase, } \\
\text { U/L }\end{array}$ & 41 & 9.1 & $21(14)$ & $21(11)$ & $21(12)$ & $21(14)$ & $20(13)$ & 1.0 \\
\hline $\begin{array}{l}\text { Lactate-dehydrogenase, } \\
\text { U/L }\end{array}$ & 41 & 1.6 & $164(18)$ & $158(22)$ & $170(21)$ & $171(20)$ & $157(17)$ & $<0.001$ \\
\hline $\begin{array}{l}\text { Alkaline-phosphatase, } \\
\text { U/L }\end{array}$ & 41 & 6.7 & $57(19)$ & $56(14)$ & $56(17)$ & $55(19)$ & $57(18)$ & 1.0 \\
\hline $\begin{array}{l}\text { Y-Glutamyl-transferase, } \\
\text { U/L }\end{array}$ & 41 & 2.7 & $15(12)$ & $14(13)$ & $14(14)$ & $14(13)$ & $14(14)$ & 1.0 \\
\hline Cholinesterase, U/L & 41 & 2.2 & $74(27)$ & $73(27)$ & $74(27)$ & $74(27)$ & $73(29)$ & 1.0 \\
\hline $\begin{array}{l}\text { Glutamate- } \\
\text { dehydrogenase, U/L }\end{array}$ & $40(1)$ & 4.8 & $2.3(1.8)$ & $2.6(2.3)$ & $2.5(2.0)$ & $2.6(1.8)$ & $2.5(1.6)$ & 1.0 \\
\hline Amylase, U/L & 41 & 4.3 & $63(21)$ & $65(21)$ & $64(21)$ & $63(21)$ & $65(20)$ & 1.0 \\
\hline Lipase, U/L & 41 & 9.7 & $32(18)$ & $33(17)$ & $33(16)$ & $33(17)$ & $32(17)$ & 1.0 \\
\hline Creatin-kinase, U/L & 41 & 2.1 & $101(90)$ & $98(92)$ & $98(92)$ & $99(92)$ & $101(94)$ & 1.0 \\
\hline
\end{tabular}


TABLE 1. Comparison of laboratory values measured in plasma for different centrifugation conditions and tube type - continued

\begin{tabular}{|c|c|c|c|c|c|c|c|c|}
\hline \multirow{3}{*}{ Parameter, unit } & \multirow{3}{*}{$\mathbf{N}^{*}$} & \multirow{3}{*}{ CV (\%) ${ }^{\dagger}$} & \multicolumn{5}{|c|}{ Centrifugation conditions per tube type } & \multirow{3}{*}{$\mathbf{P}$} \\
\hline & & & $2000 \times g / 10 \mathrm{~min}$ & $2000 \times g / 10 \mathrm{~min}$ & $3000 \times g / 7 m i n$ & $3000 \times g / 5 \mathrm{~min}$ & $3000 \times g / 5 \mathrm{~min}$ & \\
\hline & & & LiHepGel & LiHepBar & LiHepGel & LiHepGel & LiHepBar & \\
\hline Iron, $\mu \mathrm{mol} / \mathrm{L}$ & 41 & 2.4 & $17.2(8.4)$ & $17.4(8.6)$ & $17.2(8.6)$ & $17.2(8.2)$ & $17.4(8.6)$ & 1.0 \\
\hline Transferrin, $\mu \mathrm{mol} / \mathrm{L}$ & 41 & 2.0 & $32.1(6.2)$ & $32.5(6)$ & $32.6(6.3)$ & $32.1(6.3)$ & $32.3(6.8)$ & 1.0 \\
\hline $\begin{array}{l}\text { Soluble transferrin } \\
\text { receptor, } \mathrm{nmol} / \mathrm{L}\end{array}$ & 41 & 2.9 & $34.9(14.4)$ & $34.1(14)$ & $34.1(17.1)$ & $34.6(13.9)$ & $34.3(15.7)$ & 1.0 \\
\hline Ferritin, $\mu \mathrm{g} / \mathrm{L}$ & 41 & 12.2 & $80(120)$ & $80(127)$ & $80(124)$ & $81(126)$ & 79 (126) & 1.0 \\
\hline Fructosamine, $\mu \mathrm{mol} / \mathrm{L}$ & 41 & 12.7 & $256(41)$ & $261(38)$ & $256(44)$ & $258(37)$ & $264(39)$ & 1.0 \\
\hline $\begin{array}{l}\text { Free haemoglobin } \\
\text { plasma, g/L }\end{array}$ & 41 & / & $0.06(0.04)$ & $0.05(0.02)$ & $0.07(0.04)$ & $0.07(0.02)$ & $0.04(0.03)$ & $<0.001$ \\
\hline $\begin{array}{l}\text { Icterus index plasma, } \\
\text { index }\end{array}$ & 41 & / & $1(0)$ & $1(0)$ & $1(0)$ & $1(0)$ & $1(0)$ & 1.0 \\
\hline $\begin{array}{l}\text { Lipemia index plasma, } \\
\text { index }\end{array}$ & 41 & / & $10(6)$ & $8(6)$ & $9(5)$ & $12(6)$ & $11(5)$ & 1.0 \\
\hline $\begin{array}{l}\text { Creatin-kinase MB } \\
\text { immunologic, } \mu \mathrm{g} / \mathrm{L}\end{array}$ & 41 & 1.7 & $1.8(1.2)$ & $1.8(1.2)$ & $1.8(1.1)$ & $1.8(1.2)$ & $1.8(1.2)$ & 1.0 \\
\hline Myoglobin, $\mu \mathrm{g} / \mathrm{L}$ & 41 & 8.3 & $45(14)$ & $45(15)$ & $45(15)$ & $45(15)$ & $45(14)$ & 1.0 \\
\hline $\begin{array}{l}\mathrm{N} \text {-terminal pro brain } \\
\text { natriuretic peptide, pmol/L }\end{array}$ & $40(1)$ & 2.1 & $4.4(4.7)$ & $4.4(4.4)$ & $4.4(4.3)$ & $4.3(4.6)$ & $4.4(4.2)$ & 1.0 \\
\hline $\begin{array}{l}\text { Thyroid stimulating } \\
\text { hormone, } \mathrm{mU} / \mathrm{L}\end{array}$ & 41 & 5.3 & $1.96(1.17)$ & $1.91(1.15)$ & $1.97(1.12)$ & $1.92(1.2)$ & $1.95(1.13)$ & 1.0 \\
\hline $\begin{array}{l}\text { Free Triiodothyronine, } \\
\mathrm{pmol} / \mathrm{L}\end{array}$ & 41 & 6.4 & $4.7(0.6)$ & $4.6(0.6)$ & $4.6(0.7)$ & $4.6(0.7)$ & $4.6(0.6)$ & 1.0 \\
\hline Albumin, $g / L$ & 41 & 5.1 & $46(3)$ & $47(3)$ & $46(3)$ & $46(3)$ & $46(4)$ & 1.0 \\
\hline Complement C3, g/L & 41 & 7.4 & $1.1(0.2)$ & $1.1(0.2)$ & $1.1(0.2)$ & $1.1(0.2)$ & $1.1(0.2)$ & 1.0 \\
\hline Complement C4, g/L & 41 & 6.9 & $0.25(0.12)$ & $0.25(0.12)$ & $0.26(0.11)$ & $0.24(0.13)$ & $0.25(0.11)$ & 1.0 \\
\hline Alpha 1 - antitrypsin, $\mu \mathrm{mol} / \mathrm{L}$ & 41 & 4.9 & $24(5)$ & $24(5)$ & $24(5)$ & $24(5)$ & $24(5)$ & 1.0 \\
\hline Haptoglobin, g/L & 41 & $6.2 \%$ & $1.11(0.66)$ & $1.13(0.66)$ & $1.1(0.66)$ & $1.14(0.69)$ & $1.15(0.65)$ & 1.0 \\
\hline Ceruloplasmin, $\mu \mathrm{mol} / \mathrm{L}$ & 41 & $4.3 \%$ & $1.5(0.3)$ & $1.5(0.3)$ & $1.5(0.3)$ & $1.5(0.3)$ & $1.5(0.3)$ & 1.0 \\
\hline Interleukin 6, pg/mL & 41 & $1.9 \%$ & $3.8(1.5)$ & $3.6(1.3)$ & $3.5(1.3)$ & $3.6(1.3)$ & $3.6(1.6)$ & 1.0 \\
\hline
\end{tabular}

Data are presented as median and interquartile range. $\mathrm{P}<0.05$ was considered statistically significant. ${ }^{*}$ Number of individual samples used for calculation. In those parameters where this value is less than 41 , some of the samples had measured values below the measuring range of the respective assay. The number of excluded samples, where applicable, are shown in parentheses. ${ }^{\dagger} A l l$ assays were performed on a COBAS 8000 instrument (Roche Diagnostics) using only proprietary reagents. CVs of day-to-day QC control values of the respective parameter. LiHepGel - Lithium Heparin plasma from tubes with gel separator. LiHepBar - Lithium Heparin plasma from tubes with mechanical separator. CV - coefficient of variation.

\section{Statistical analysis}

Linear mixed effect modelling with BenjaminiHochberg multiple testing correction was performed for different centrifugation conditions (fixed effects for centrifugation condition and sex, random effect for replicate). As a post-hoc test for our linear mixed effects modelling we calculated pairwise differences of least squares means for the fixed effects of interest. For the parameters with statistically significant differences, agreement analyses using the total allowable error (derived from the Ricos criteria on biological variation) as cut-off for the total deviation index (TDI) and tar- 
geting at a coverage probability (CP) of $80 \%$ were performed as suggested by Barnhart et al. $(24,25)$. Normality testing was done using the Anderson Darling test. All statistical analyses were performed with SPSS 23.0 (SPSS Inc. Chicago, USA), R v.3.4.1 (The R Foundation, Vienna, Austria) and python scripts (Python Software Foundation, Python Language Reference, version 3.6). A-priori sample size calculation was performed using G-Power V3.1.9.2 (Heinrich-Heine-University, Düsseldorf, Germany) yielding a statistical power of 0.8 (26).

\section{Results}

Measurements of C-reactive protein (CRP), lipoprotein a (Lp(a)), N-terminal pro brain natriuretic pep- tide (NT-proBNP), glutamate-dehydrogenase (GLDH), anti-streptolysin O (ASL), anti-Müller hormone (AMH), carcinoembryonic antigen (CEA) and alpha fetoprotein (AFP) yielded values below the measuring range in some samples. Subsequently, respective samples were not used for further calculations. Since samples were collected in a healthy group of volunteers, high sensitive Troponin $\mathrm{T}$ (hsTnT), pregnancy-associated plasma protein A (PAPP-A), ß human chorionic gonadotropin (BHCG) and rheumatoid factor (RF) were below the measuring range in over $50 \%$ of study participants samples and were therefore excluded from calculations. For all other parameters, values within the measuring range could be obtained in samples of all 41 subjects (Table 1 for analytes measured in

TABLE 2. Comparison of laboratory values measured in serum for different centrifugation conditions

\begin{tabular}{|c|c|c|c|c|c|c|}
\hline \multirow{2}{*}{ Parameter } & \multirow{2}{*}{$\mathbf{N}^{*}$} & \multirow{2}{*}{ CV (\%) ${ }^{\dagger}$} & \multicolumn{3}{|c|}{ Centrifugation conditions } & \multirow{2}{*}{$\mathbf{P}$} \\
\hline & & & $2000 \times g / 10 \mathrm{~min}$ & $3000 \times g / 7 \mathrm{~min}$ & $3000 \times g / 5 \mathrm{~min}$ & \\
\hline Free haemoglobin, g/L & 41 & / & $0.05(0.03)$ & $0.07(0.04)$ & $0.07(0.03)$ & 0.001 \\
\hline Icterus index, index & 41 & / & $1(0)$ & $1(0)$ & $1(0)$ & 0.99 \\
\hline Lipemia index serum, index & 41 & / & $5(4)$ & $6(5)$ & $6(5)$ & 0.99 \\
\hline Anti-Müllerian hormone, pmol/L & $37(4)$ & 4.4 & $25.7(23.5)$ & $25.7(23.5)$ & $25.7(23.5)$ & 0.99 \\
\hline Prealbumin, $\mu \mathrm{mol} / \mathrm{L}$ & 41 & 5.7 & $4.8(1.4)$ & $4.9(1.1)$ & $4.8(1.5)$ & 0.99 \\
\hline Carcinoembryonic antigen, U/L & $38(3)$ & 10.2 & $23.7(16.1)$ & $23.7(14.8)$ & $23.7(14.8)$ & 0.99 \\
\hline Alpha Fetoprotein, U/L & $38(3)$ & 7.4 & $19.9(13.1)$ & $19.9(14.5)$ & $19.9(12.9)$ & 0.99 \\
\hline Cytokeratin fragment $21-1, \mu \mathrm{g} / \mathrm{L}$ & 41 & 6.1 & $1.2(0.7)$ & $1.2(0.8)$ & $1.2(0.7)$ & 0.99 \\
\hline Cancer-Antigen 125, U/L & 41 & 5.7 & $120(60)$ & $120(80)$ & $120(70)$ & 0.99 \\
\hline Cancer-Antigen19-9, U/L & 41 & 6.2 & $70(150)$ & $70(150)$ & $60(150)$ & 0.99 \\
\hline Cancer-Antigen 15-3, U/L & 41 & 6.4 & $170(110)$ & $170(100)$ & $170(110)$ & 0.99 \\
\hline S100 protein, $\mu \mathrm{g} / \mathrm{L}$ & 41 & 7.2 & $0.05(0.02)$ & $0.05(0.02)$ & $0.05(0.02)$ & 0.99 \\
\hline Neuron specific enolase, $\mu \mathrm{g} / \mathrm{L}$ & 41 & 6.2 & $11(3)$ & $12(3)$ & $12(3)$ & 0.71 \\
\hline Cancer-Antigen 72-4, U/L & 41 & 6.5 & $13(37)$ & $13(36)$ & $13(37)$ & 0.99 \\
\hline Cystatin C, nmol/L & 41 & 2.9 & $62.9(11.2)$ & $62.9(12)$ & $62.9(12.7)$ & 0.99 \\
\hline $\begin{array}{l}\text { Cystatin C estimated glomerular filtration rate, } \mathrm{mL} / \\
\mathrm{min} / 1.73 \mathrm{~m}^{2}\end{array}$ & 41 & / & $97(19)$ & $99(19)$ & $98(20)$ & 0.99 \\
\hline Soluble fms-like tyrosine kinase- $1, \mathrm{pg} / \mathrm{mL}$ & 41 & 16.0 & $89.4(11.7)$ & $89.9(11)$ & $88.8(13.1)$ & 0.99 \\
\hline Placental growth factor, $\mathrm{pg} / \mathrm{mL}$ & 41 & 16.8 & $14.89(4.5)$ & $14.64(4.2)$ & $14.95(4.2)$ & 0.99 \\
\hline sFlt1/PIGF Ratio, ratio & 41 & / & $6.29(1.6)$ & $6.4(1.6)$ & $6.4(1.6)$ & 0.99 \\
\hline
\end{tabular}

Data are presented as median and interquartile range. $\mathrm{P}<0.05$ was considered statistically significant. *Amount of individual samples used for calculation. In those parameters where this value is less than 41 , some of the samples had measured values below the measuring range of the respective assay. The number of excluded samples, where applicable, are shown in parentheses. ${ }^{\dagger}$ All assays were performed on a COBAS 8000 instrument (Roche Diagnostics) using only proprietary reagents. CVs of day-to-day QC control values of the respective parameter. CV - coefficient of variation. 
plasma and Table 2 for analytes measured in serum). As some of the parameter values were normally distributed while others were not, we chose to depicture all values as median and interquartile range (IQR).

Almost no parameters showed any significant differences after overall multiple testing corrections. Only lactate-dehydrogenase (LD) and measurements of $\mathrm{fHb}$ in serum ( $\mathrm{fHb}$ _S ) and plasma ( $\mathrm{fHb}$ _P) showed respective overall multiple testing-corrected P-values of less than 0.05 (Table 1 and Table 2). A more detailed analysis of LD results revealed that the observed differences in measurements originated from the tube types (LiHepGel and LiHepBar) irrespective of the centrifugation conditions (Figure 2A). Bicarbonate (BIC) measurements, which were non-significant after overall multipletesting correction ( $P=0.286$ ), also showed slight yet significant differences when comparing tube types (Figure 2B). Measurement of $\mathrm{fHb}$. $\mathrm{P}$ and $\mathrm{fHb}$ _S differed between tube types and also between centrifugation settings (Figure $2 \mathrm{C}$ and 2D). When testing for clinical significance of these differences using the agreement analysis and a cutoff of $11.4 \%$ (total allowable error according to Ricos criteria) for LD, the coverage probability to get the same result in LiHepBar vs LiHepGel tubes was $\sim 60 \%$ and $\sim 50 \%$ for centrifugation at $2000 \mathrm{xg}$ for 10 minutes and $3000 x g$ for 5 minutes, respectively. This means that in both cases we did not get an agreement between the measurements (the probability of values differing between these tubes by more than $11.4 \%$ at the same centrifugation setting is greater than 40\%) and differences in LD measurements between LiHepGel and LiHepBar tubes are most probably to be considered clinically significant.

\section{Discussion}

In our study we could show that apart from $\mathrm{fHb}$, none of the 77 analytes investigated differed significantly between centrifugation settings (Table 1, Table 2 and Figure 2). Looking more closely into these $\mathrm{fHb}$ differences we found that the higher centrifugation speed of $3000 \mathrm{xg}$ is associated with higher $\mathrm{fHb}$ concentrations (Figure $2 \mathrm{C}$ comparison
3-5 and figure 2D comparisons 6-8), as already mentioned by Lippi et al. (1). However, when reducing the time of centrifugation to 5 minutes also at $3000 \times$ g, these significances were diminished (Figure 2D comparisons 6-7) or even vanished (Figure $2 \mathrm{C}$ comparison $3-4)$. As these differences in $\mathrm{fHb}$ values between centrifugation settings were rather small and did not seem to have any influence on the tested clinical chemistry analytes, our findings suggest that respective samples for these analyses can be centrifuged at 3000xg for 7 or 5 minutes without a significant alteration of test results. However, a raise in $\mathrm{fHb}$ even on a low level might have impact on analytical outcome if this increase contributes to a final $\mathrm{fHb}$ concentration above an analyte specific haemolysis cut-off, subsequently leading to a deletion of the respective result.

In order to compare our results to other studies, we scanned the literature for evidence based recommendations and found these to be very scarce regarding clinical chemical analytes, finding only expert opinion suggestions ranging from 10 to 20 minutes and 1500 to $3000 \mathrm{xg}$ at 15 to $25^{\circ} \mathrm{C}$, respectively (6-10). For other parameters such as coagulation analyses however, several studies have been undertaken aiming at reducing centrifugation time at higher g-forces $(3,4,17,18,27,28)$. Considering the potential analytical consequences, it seems odd that no real standardization has been achieved for this step while the analytical processes are constantly improved and accurately controlled. However, there was one investigation carried out by Møller et al. in which nine selected parameters as well as haemolysis, lipemia and icterus index were measured in serum and lithium heparin sample pairs from 40 patients. Each of these sample pairs were centrifuged at $2200 x g$ for 10 minutes and at 3000xg for 5 minutes (19). Similar to our findings, the authors could show a good correlation of analyses. However, conflicting with our data, they found LD measurements to increase by $6.3 \%$ on average at higher centrifugation speed. Maybe the difference in study population contributed to this fact, as the authors collected blood from hospitalized patients whereas we drew blood from healthy volunteers with lower LD rang- 

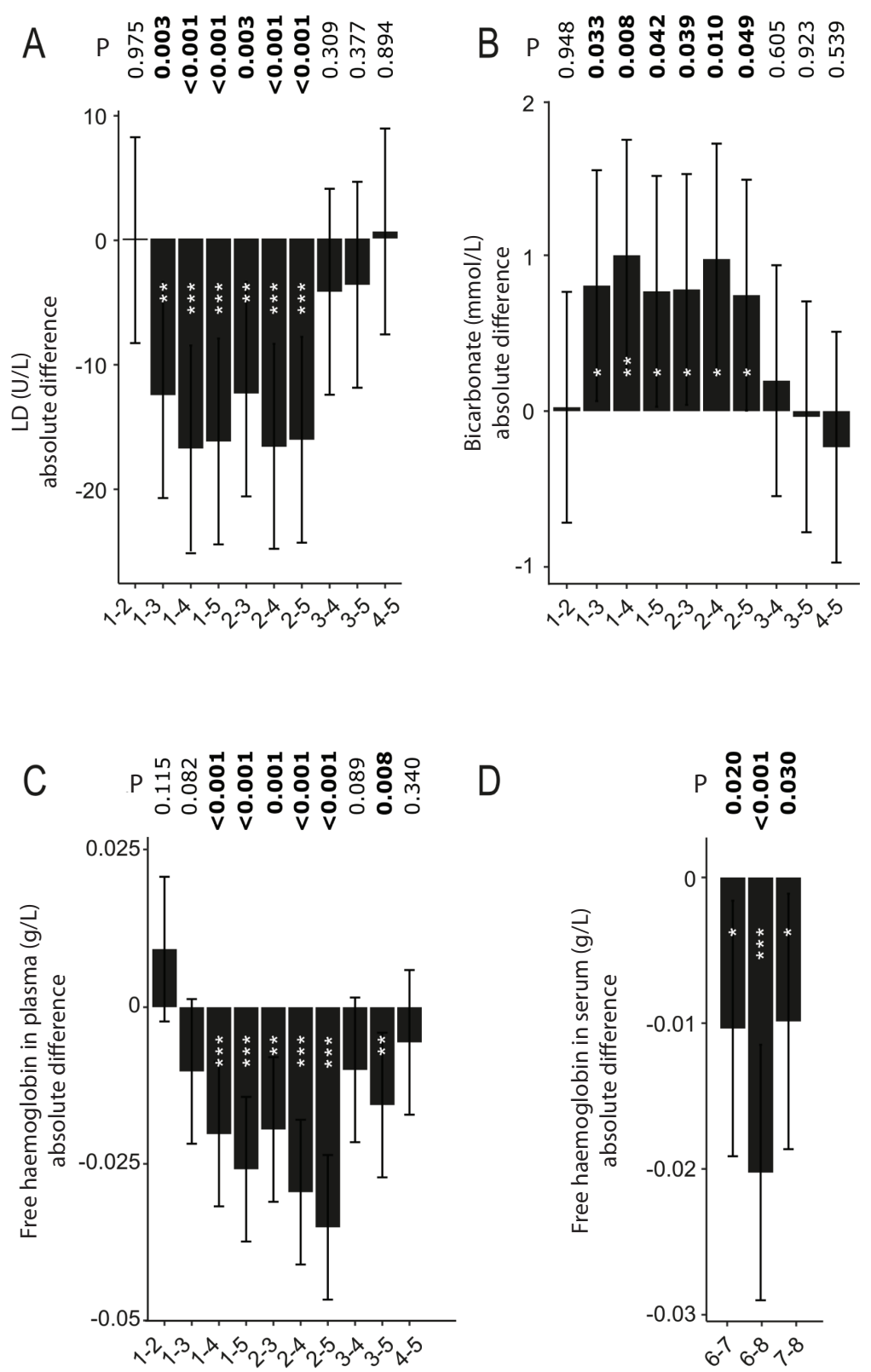

Figure 2. Comparison of absolute results of lactate-dehydrogenase (A), bicarbonate (B), free haemoglobin in plasma (C) and serum (D) between centrifugation settings and tube types. Scales on the $y$-axis represent differences in absolute values of the respective analyte. IDs on the x-axis represent the different centrifugations settings: 1 - LiHepBar 2000xg/10min; 2 - LiHepBar 3000xg/5min; 3 - LiHepGel 2000/10min; 4 - LiHepGel 3000xg/5min; 5 - LiHepGel 3000xg/7min; 6 - Serum 2000xg/10min; 7 - Serum 3000xg/5min; 8 - Serum 3000xg/7min. ${ }^{* P}<0.05 ;{ }^{*} \mathrm{P}<0.01 ;{ }^{* *} \mathrm{P}<0.001$. P-values $<0.05$ were considered statistcally significant.

es. Also the analytical platform differs as we performed analyses on a Roche COBAS system and Møller et al. used an Abbott Architect system, in which haemolysis is reported in levels rather than as quantitative value (29). A statistical evaluation of these levels therefore may differ from ours.
In combination with findings of Sedille-Mostafaie et al. demonstrating that blood for coagulation testing can be centrifuged at 3000xg for 7 minutes without altering measurement values in a broad variety of haemolysis analytes, samples for clinical chemistry and coagulation analyses could eventu- 
ally be centrifuged simultaneously (16). As many laboratories process samples using automated processes, combining pre-analytical and analytical procedures, this could help reducing intra-laboratory TAT as well as the number of centrifuges needed.

Besides these findings we also evaluated the newly introduced BD Barricor tubes (LiHepBar), which use a mechanical element to separate plasma from cellular components during centrifugation. Comparing measurements between centrifugation settings in these tubes, we did not find a significant difference in any of the tested analytes, including $\mathrm{fHb}$. However, comparing analysis results between LiHepBar and the commonly used LiHepGel tubes, we found differences in $\mathrm{fHb}, \mathrm{LDH}$ and $\mathrm{BIC}$ measurements, performed in plasma retrieved from identical centrifugation settings (Figure $2 \mathrm{~A}-\mathrm{C}$ ). Since the mechanical separator within the LiHepBar tubes allows cellular blood components to pass during the entire centrifugation process and gel separators in the respective tubes occlude this passage already at an early stage of centrifugation, we hypothesized that differences in residual cell count may be the reason for the disagreement in $\mathrm{fHb}$ and $\mathrm{LDH}$ values between these two tubes. In a small explorative experiment we could strengthen our assumption that LDH levels may be increased upon cellular release (data not shown), however, a separate study would be needed to conclusively prove this causal relationship. Nevertheless, according to our data, separate $\mathrm{LDH}$ reference values for LiHepBar tubes appear to be necessary.

For the minor, yet significant BIC differences of about $1 \mathrm{mmol} / \mathrm{L}$ between these tubes we could not find a definite reason. Although respective

\section{References}

1. Lippi G, Blanckaert N, Bonini P, Green S, Kitchen S, Palicka V, et al. Haemolysis: An overview of the leading cause of unsuitable specimens in clinical laboratories. Clin Chem Lab Med 2008;46:764-72. https://doi.org/10.1515/CCLM.2008.170

2. Guder W, Narayanan S, Wisser H, Zawta B, eds. What has to be done on specimen arrival? - specimen processing, centrifugation, distribution. In: Guder W, Narayanan S, Wisser H, samples in this study were treated identically, we assume that the exposure to air in these tubes or differences in proceeding cellular metabolism might be contributing to this fact. However, as we could not find similar results in current literature, we were not able to confirm or compare our findings.

As limitation to this study, we want to mention the fact that analyses, which are only increased in certain medical conditions (hsTnT, RF, BHCG, PAPP-A) could not be investigated properly, since only healthy volunteers were tested. Subsequently, we could also not cover specific medical conditions possibly affecting sample stability in different centrifugation conditions.

In conclusion, in this study, we provide evidence that samples for the measurement of 77 clinical chemistry analytes may be centrifuged at 3000xg for 7 or 5 minutes without any impact on test results compared to centrifugation at 2000xg for 10 minutes, yielding a benefit of up to 5 minutes in intra-laboratory TAT. As residual leucocyte count, $\mathrm{fHb}$ and subsequently lactate dehydrogenase results are lower in the BD Barricor tubes, a new LDH reference range might be necessary when using these tubes.

\section{Acknowledgments}

We want to thank Roche Diagnostics for funding this research by providing the necessary assays for this study.

\section{Potential conflict of interest}

Janne Cadamuro received a personal fee as guest member of a Becton Dickinson Advisory board meeting.

Zawta B eds. Diagnostic samples: From the patient to the laboratory. 4th ed. Weinheim: Wiley, 2009. p. 44-5.

3. Lippi G, Salvagno GL, Montagnana M, Manzato F, Guidi GC. Influence of the centrifuge time of primary plasma tubes on routine coagulation testing. Blood Coagul Fibrinolysis 2007;18:525-8. https://doi.org/10.1097/ MBC.0b013e3281eec945 
4. Lippi G, Salvagno GL, Montagnana M, Poli G, Guidi GC. Influence of centrifuge temperature on routine coagulation testing. Clin Chem 2006;52:537-8. https://doi.org/10.1373/ clinchem.2005.063149

5. Lesche D, Geyer R, Lienhard D, Nakas CT, Diserens G, Vermathen $P$, Leichtle AB. Does centrifugation matter? Centrifugal force and spinning time alter the plasma metabolome. Metabolomics 2016;12:159. https://doi.org/10.1007/ s11306-016-1109-3

6. Clinical and Laboratory Standards Institute (CLSI). Collection, transport, and processing of blood specimens for testing plasma-based coagulation assays and molecular hemostasis assays. Approved Guideline - Fifth edition. CLSI document H21-A5. Wayne (PA): CLSI, 2008.

7. Adcock DM, ed. Sample integrity and preanalytical variables. In: Kitchen S, Olson JD, Preston EE, eds. Quality in laboratory hemostasis and thrombosis. 1st ed. Chichester: Wiley-Blackwell, 2009. p. 31-42. https://doi. org/10.1002/9781444303575.ch4

8. Guder W, Fiedler GM, da Fonseca-Wollheim F, Schmitt $Y$, Töpfer $G$, Wisser H, Zawta B. eds. The quality of diagnostic samples. Recommendations of the Working Group on Preanalytical Quality of the German United Society for Clinical Chemistry and Laboratory Medicine. 4th ed. Munich, DGKL, 2015.

9. Hagemann P. [Präanalytische phase]. In: [Labor und Diagnose]. Thomas L, ed. 7th ed. Frankfurt: TH Books, 2008. p. 1965-74. (in German)

10. German Institute for Standardization (DIN). Haemostaseology - Blood collection - Part 1: Preparation of plasma from citrated venous blood for coagulation testing. Standard 58905-1. Berlin: DIN, 2000.

11. Venous blood collection - FAQ. Becton Dickinson. Available at: http://www.bd.com/vacutainer/faqs/\#venous_faq. Accessed April 19th 2017.

12. Vacuette Preanalytics Manual. Greiner BioOne. [Electronic version] Available at: https://www.gbo.com/fileadmin/user_upload/Downloads/Brochures/Brochures_ Preanalytics/English/980183_Preanalytics_Manual_e_ rev05_0216_lowres.pdf. Accessed April 25th 2017.

13. Centrifugation recommendations for S-monovette ${ }^{\oplus}$. Sarstedt. [Electronic version] Available at: https://www.sarstedt.com/fileadmin/user_upload/99_Gebrauchsanweisungen/US/427_a_s_monovette_empf_zentri_USA_0610. pdf. Accessed April 25th 2017.

14. Roberts $T$, Smith $M$, Roberts B. Observations on centrifugation: Application to centrifuge development. Clin Chem 1999;45:1889-97.

15. Clinical and Laboratory Standards Institute (CLSI). Procedures for the handling and processing of blood specimens for common laboratory tests. Approved guideline - Fourth edition. CLSI document H18-A4. Wayne (PA): CLSI, 2010.

16. Sedille-Mostafaie N, Engler H, Lutz S, Korte W. Advancing haemostasis automation--successful implementation of robotic centrifugation and sample processing in a tertiary service hospital. Clin Chem Lab Med 2013;51:1273-8. https:// doi.org/10.1515/cclm-2012-0625
17. Boissier E, Sevin-Allouet M, Le Thuaut A, De Gaalon S, Trossaert $M$, Rozec B, et al. A 2-min at $4500 \mathrm{~g}$ rather than a 15min at $2200 \mathrm{~g}$ centrifugation does not impact the reliability of 10 critical coagulation assays. Clin Chem Lab Med 2017;55:e118-e21. https://doi.org/10.1515/cclm-2016-0670

18. Suchsland J, Friedrich N, Grotevendt A, Kallner A, Ludemann $J$, Nauck M, Petersmann A. Optimizing centrifugation of coagulation samples in laboratory automation. Clin Chem Lab Med 2014;52:1187-91. https://doi.org/10.1515/cclm2014-0038

19. Møller MF, Søndergaard TR, Kristensen HT, Münster AB. Evaluation of reduced centrifugation time and higher centrifugal force on chemistry and immunochemistry analytes in plasma and serum. Ann Clin Biochem 2017,54:593-600.

20. Clinical and Laboratory Standards Institute (CLSI). Procedures for the collection of diagnostic blood specimens by venipuncture. Approved Standard - Sixth Edition. CLSI document GP41-A6. Wayne (PA): CLSI, 2007.

21. Guder WG, Narayanan S. Sample transport, treatment after arrival, storage and disposal. In: Guder WG, Narayanan S, eds. Pre-examination procedures in laboratory diagnostics. Berlin: De Gruyter, 2015. p251-264.

22. Petrova DT, Cocisiu GA, Eberle C, Rhode KH, Brandhorst $G$, Walson PD, Oellerich M. Can the Roche hemolysis index be used for automated determination of cell-free hemoglobin? A comparison to photometric assays. Clin Biochem 2013;46:1298-301. https://doi.org/10.1016/j.clinbiochem.2013.06.018

23. Unger J, Filippi G, Patsch W. Measurements of free hemoglobin and hemolysis index: Edta- or lithium-heparinate plasma? Clin Chem 2007;53:1717-8. https://doi.org/10.1373/ clinchem.2007.091421

24. Ricos C, Alvarez V, Cava F, Garcia-Lario JV, Hernandez A, Jimenez $C V$, et al. Current databases on biological variation: Pros, cons and progress. Scand J Clin Lab Inv 1999;59:491500. https://doi.org/10.1080/00365519950185229

25. Barnhart HX, Yow E, Crowley AL, Daubert MA, Rabineau D, Bigelow $R$, et al. Choice of agreement indices for assessing and improving measurement reproducibility in a core laboratory setting. Stat Methods Med Res 2016;25:2939-58. https://doi.org/10.1177/0962280214534651

26. Faul F, Erdfelder E, Lang A-G, Buchner A. A flexible statistical power analysis program for the social, behavioral, and biomedical sciences. Behavior Research Methods 2007;39:17591. https://doi.org/10.3758/BF03193146

27. Chandler WL, Ferrell C, Trimble S, Moody S. Development of a rapid emergency hemorrhage panel. Transfusion 2010;50:2547-52. https://doi.org/10.1111/j.15372995.2010.02753.x

28. Kao CH, Shu LC, Yen WH. Evaluation of a high-speed centrifuge with rapid preparation of plasma for coagulation testing to improve turnaround time. J Biomed Lab Sci 2010;22:23-7.

29. Dolci A, Panteghini M. Harmonization of automated hemolysis index assessment and use: Is it possible? Clin Chim Acta 2014;432:38-43. https://doi.org/10.1016/j.cca.2013.10.012 